\title{
EL PUEBLO DE ZARAGOZA EN PALMARES Y LA FRAGUA DE UNA IDENTIDAD DESDE EL SIGLO XIX
}

Recibido: $29-08-2016$

Aprobado: 22-10-2016

\author{
Javier Rodríguez Sancho \\ Universidad de Costa Rica, Sede de Occidente
}

\begin{abstract}
Javier Rodríguez Sancho es historiador de la Sección de Historia y Geografía, también del Sistema de Estudios Generales. Además, encargado de la Sección de Colecciones Patrimoniales del Museo Regional de San Ramón de la Universidad de Costa Rica.
\end{abstract}

\section{Resumen}

\begin{abstract}
En el artículo se interpreta un fenómeno histórico acaecido en Palmares en la provincia de Alajuela, que desde el siglo XIX ya era visible, según las fuentes históricas. Asimismo, se muestra un conflicto y sus vicisitudes entre el pueblo de Zaragoza de Palmares con el cura párroco a raíz del proyecto de construcción del Santuario de Nuestra Señora del Pilar. Además, examina las relaciones históricas entre las comunidades de la Región de Occidente de Costa Rica desde el siglo XIX.
\end{abstract}

Palabras clave: Región de Occidente, Zaragoza de Palmares, historia local, conflicto e identidad comunal

\begin{abstract}
In this article a historical phenomenon occurred in Palmares in the province of Alajuela, which since the nineteenth century it was already visible, according to historical sources is interpreted. It also shows a conflict and its vicissitudes between the town of Zaragoza of Palmares with the parish priest in the wake of the project construction of the sanctuary of Our Lady of Pilar. It also examines, the historical relations between the communities in the Western region of Costa Rica from the nineteenth century.
\end{abstract}

Keywords: Western region, Zaragoza of Palmares, local history, conflict and communal identity.

\section{LOS COLONIZADORES EN LA REGIÓN DE OCCIDENTE}

El proceso colonizador que propició el poblamiento de la región occidental en la provincia de Alajuela, dio inicio en la primera mitad del siglo XIX, un fenómeno explicado por las historiadoras Miriam Pineda y Silvia Castro (2012). Las fuentes históricas confirman un éxodo en el que participaron pequeños grupos de familias, que inmigraron paulatinamente provenientes de Alajuela o Heredia y que a través de Atenas ingresaron hasta el otrora Valle de los Palmares. Sin dejar de referir, que villas como las de Grecia y Naranjo, también recibieron migrantes de dichos centros poblacionales y en menor medida, de San José, tal como lo expone José Luis Torres (2007). 
Antes del arribo de estos colonos, la región estuvo habitada por tribus dispersas de acuerdo con el hallazgo de vestigios cerámicos, piedras labradas, caminos y de restos óseos humanos (Vásquez, Fallas \& Jiménez, 2014). Se conoce que el cacicazgo de Garabito, se extendió desde el litoral Pacífico hasta la actual Zona Norte de Costa Rica (Badilla \& Solórzano, 2010, pp.31 y 32).

En la segunda mitad del siglo XIX, Atenas, Palmares y San Ramón mantuvieron estrechos vínculos en aspectos de índole familiar, comercial, productivo, político y eclesiástico. En términos administrativos y jurisdiccionales de la Iglesia católica, Atenas era una Filial en 1846, mientras, San Ramón fue una Ayuda de Parroquia, que en 1854 adquirió el rango de Parroquia bajo el patronazgo de San Ramón Nonato; cabe agregar que conformaban la jurisdicción respectiva los caseríos de San Juan, San Isidro y Las Mercedes _Palmares_. Sin embargo, la ermita de este último lugar, fue erigida como filial en 1866 al mando del teniente cura Rafael de Jesús Soto, primer sacerdote designado en el lugar por el obispo Anselmo Llorente y La Fuente. En ese espacio y de acuerdo con un conteo efectuado por los mismos moradores del barrio, 1452 personas lo habitaban, una suma que fue contraria al impreciso Censo Nacional que solo registró a 750 (Pérez \& González, 1993). No es arriesgado suponer, que los vecinos se conocían entre sí y que no quisieron pasar desapercibidos en medio de sus propios conteos de cara al universo que iban forjando. En el Mapa 1, se muestra la extensión territorial de la región de Occidente en 1883, que lindaba con la frontera norte de Costa Rica, al menos, Grecia y Naranjo tuvieron esa particularidad geográfica.

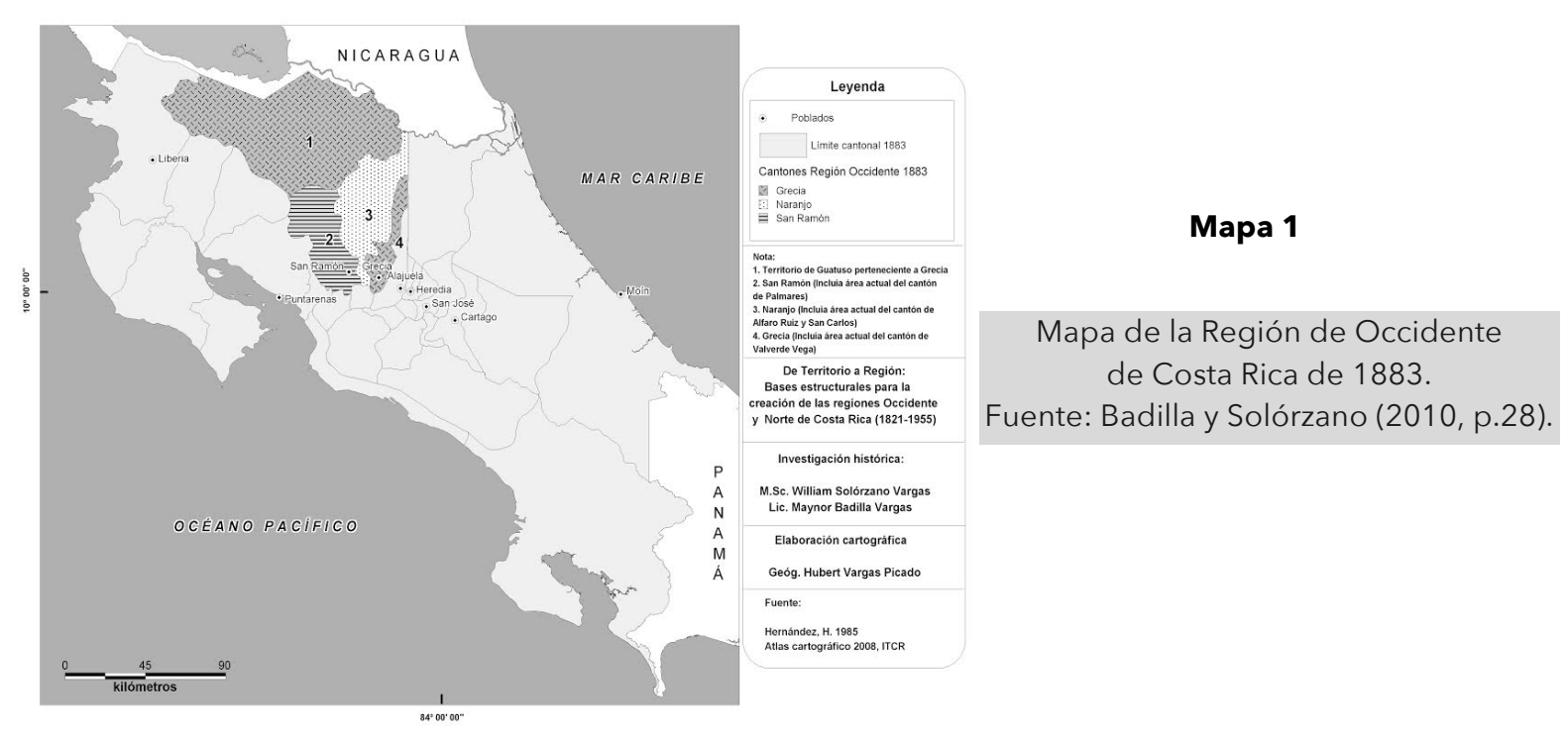


En el Primer Sínodo Diocesano convocado en agosto de 1881 por el recién electo monseñor Bernardo Augusto Thiel ${ }^{1}$, se le otorgó el rango de parroquia a las filiales del territorio costarricense, lo que favoreció a la de San Anselmo de los Palmares. Para finales del siglo XIX, la Iglesia católica contaba con 58 parroquias y cerca de 154 templos construidos, que sin duda, consolidaron las tareas de la evangelización entre las poblaciones que atendieron, tal como lo concebía monseñor Thiel (1900), adquiriendo una cuota de poder político, que junto a su investidura simbólica y el dictado de ciertas normas morales a gusto, les confirió un marcado control social que lograron sostener por décadas, según lo esclarece Alfonso González (1997).

En ese contexto, la década de 1880 estuvo marcada por un ácido enfrentamiento entre la jerarquía eclesiástica y un sector liberal encabezado por el presidente Próspero Fernández a partir de 1882. Los entretelones de las disputas por el poder decantaron en la expulsión del obispo Thiel con varios sacerdotes, en apego a las Ilamadas leyes anticlericales, que se materializaron con la fuerza de las circunstancias en $1884{ }^{2}$ un fenómeno estudiado con lujo de detalle por el historiador Claudio Vargas (2015).

El conflicto referido que se prolongaría en el tiempo, propició saldos negativos en cuanto a los alcances y a la influencia del clero en las estructuras de poder local y regional. El sacerdote Manuel Bernardo Gómez Salazar_años después nombrado en Las Mercedes_fue parte de una lista de los desterrados hacia el inhóspito Guatuso, luego a Nicaragua. La designación de Gómez en la villa de Palmares en abril de 1897, estuvo condicionada por la necesidad de no exponerlo ante ciertos líos políticos en que se había involucrado y sobre todo, por las opiniones en contra de uno de los más conspicuos dirigentes

\footnotetext{
${ }^{1}$ Este obispo arribó a Costa Rica para ayudar a solventar los problemas administrativos de la misma Iglesia católica como la llamada vacante episcopal, que entre 1871 y 1880 no contó con un obispo titular. Su episcopado comenzó en setiembre de 1880 en el que fundó El Mensajero del Clero en 1882, El Eco Católico en 1883 y La Unión Católica en 1890, además, creó la Biblioteca Episcopal, ordenó y enriqueció el Archivo Histórica de la Curia. En poco más de dos décadas emitió 48 cartas pastorales, 123 circulares generales y 21 para el clero. El episcopado de Thiel, se cerró con su muerte en setiembre de 1901.

${ }^{2}$ Entre otras acciones, se operó la secularización de los cementerios, el matrimonio civil y la aparición posterior del Registro Civil, que contrarrestaban el poderío histórico de la Iglesia católica, una protagonista indiscutible desde el siglo XVI hasta comienzos del XIX. También, se promovió la educación laica de orientación positivista, la prohibición de las órdenes religiosas y la expulsión de los jesuitas (Cedeño Castro, 2004, p. 213).
} 
liberales, el señor Rafael Iglesias. En un libro parroquial, Gómez dejó constancia del acontecimiento con su puño y letra: "salí desterrado a Guatuso por haber combatido la reelección de Rafael Iglesias..." (Parroquia de la Merced de Palmares, 1904, s.p).

Por si eso fuera poco, en un plebiscito regional en noviembre de 1916, Gómez jugó un protagonismo nada despreciable para que San Ramón no se convirtiera en la provincia de La Paz, al menos, 746 palmareños votaron en contra de ese proyecto y 302 apoyaron la iniciativa ramonense. Los intereses de la Municipalidad de Alajuela fueron defendidos por Gómez, tal como lo puntualiza el historiador Carlos Abarca (1999). Como un testigo directo de ello, el Parque Palmares en la ciudad de Alajuela, es una evidencia concreta de aquel conflicto regional, que enemistó a comunidades de la zona rural costarricense y que no estuvo ajena a las disputas entre los curas y un sector liberal (Vargas, 2015).

\section{LA DISPUTA Y UN NOMBRE SIMBÓLICO EN PALMARES}

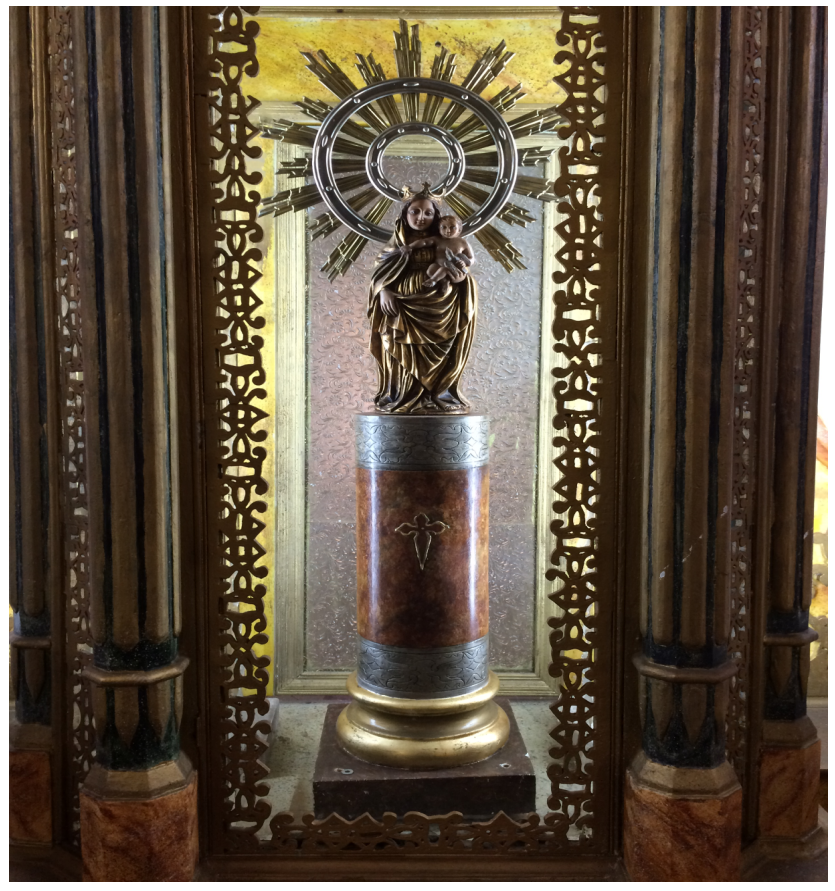

Foto 1 Réplica de la imagen del Pilar de Zaragoza

en España enviada en 1960.
A finales del siglo XIX, el sacerdote Esteban Sigifredo Echeverri emplazado en Las Mercedes, bautizó algunos de los conjuntos de ranchos de la parroquia, entre ellos, el situado en el camino real en dirección hacia el sur. El enlace carretero entre Palmares y Atenas, era estratégico ya que Atenas fue un centro importante para la región de Occidente al ubicarse próximo a la llamada Carretera Nacional, una estratégica ruta para carretas de bueyes, que enlazó a San José con Puntarenas desde la década de 1840 de acuerdo con Javier Rodríguez Sancho (2014). Es 
probable que la referida designación del Padre Echeverri, le otorgara una credencial ficticia a una ranchería sin nombre denominada desde ese momento como Zaragoza y esta buscaría apropiarse del fastuoso título con el paso del tiempo. Al calor de los acontecimientos, no sobra decir, que Palmares había adquirido el cantonato en 1888, una acreditación de orden civil, pese a conservar la misma jurisdicción territorial de la parroquia, una práctica similar que aconteció en un sin número de localidades en las apreciaciones del historiador Marco Aurelio Sandí Morales (2011, pp.53-99).

En Zaragoza, se fueron amasando unos legados y fondos, a saber, una casa donada para usufructuar en alquiler y unos dineros ofrecidos por los moradores, que apuntaban al financiamiento de la construcción del Santuario de Nuestra Señora del Pilar, advocación similar a la de Zaragoza, ciudad principal en la provincia de Aragón en España (Rodríguez Sancho, 2007). Sin embargo, los trabajos en el templo parroquial, que iniciaron en abril de 1894 con la colocación de la primera piedra por parte del arzobispo de Guatemala desterrado en Costa Rica, monseñor Ricardo Casanova Estrada (Cal, 2005) y con el posterior arribo del padre Gómez en abril de 1897, esta obra priorizó el esfuerzo económico, el constructivo y el logístico de la feligresía local (Sanou, 2001). En la mentalidad del Padre Gómez, los vecinos tenían que concentrarse en lo relevante, evitando así ciertas distracciones de menor envergadura como una simple ermita a escasos kilómetro y medio de distancia (Rodríguez Sancho, 2007). Ante este dilema, surge un asunto que acarrea algunas preguntas en el presente en razón de las listas de los donantes, del voluntariado o

\footnotetext{
${ }^{3}$ Se ha dicho que el clérigo guatemalteco aprovechó el discreto acto en Palmares del 22 de abril de 1894 para lanzar acusaciones en contra del dictador Justo Rufino Barrios, también denunció la persecución del clero que lo afectaba directamente (Cedeño Castro, 2004, pp. 207-222). Según Fabián Madrigal, Casanova Estrada habló en contra de Manuel Lisandro Barillas Bercián, quien fue el que lo exilió. Sin embargo, Justo Rufino Barrios murió en 1885 y en la documentación existente, no se relaciona con el exilio de Casanova. El general José María Reyna Barrios decretó en 1897, tras la muerte de Barillas Bercián en 1896, una amnistía que le permitió a Casanova volver a Guatemala en 1897. El complejo conflicto de finales del siglo XIX, también se protagonizó en otras sociedades de América Latina con consecuencias negativas para la Iglesia católica. Es importante agregar, que hubo curas de orientación liberal, masones y rebeldes que fueron un dolor de cabeza, además, otros con escasa formación académica, un asunto que Thiel intentó corregir desde su arribo en 1880. La indisciplina, las contradicciones pastorales y el mal comportamiento de algunos curas, entre líos amorosos, herencias, problemas políticos o de otra índole, los llevó a los tribunales, según lo comprueba el estudio de Alfonso González Ortega (1997). En algunas situaciones, los obispos trasladaron de las parroquias a los curas en problemas legales para contrarrestar los escándalos públicos.
} 
de los boyeros del caserío de Zaragoza, ya que sobresalen en número, por tanto, ¿podría interpretarse como una evidencia de un acercamiento con Gómez? o ¿era una forma de persuadirlo para que consintiera otra edificación? Los registros parroquiales de puño y letra de Gómez así lo dejan ver, entre estos, los libros de actas de la Junta Edificadora de la Parroquia de la Merced. Además, las listas por labores ejecutadas -turnadamente según el caserío al que se perteneciera- como la de boyeros, mano de obra u otras, en donde los residentes de Zaragoza mantuvieron un protagonismo en comparación con otros barrios, más allá del central. (Parroquia de la Merced de Palmares, 1894-1914, s.p).

De acuerdo con una documentación consultada en el Archivo Histórico Arquidiocesano de la Curia Metropolitana de San José (1906), el señor cura había recogido unos fondos propiedad del Pilar, no obstante, encontró una férrea resistencia por parte de los zaragozanos, quienes apelaron al primer obispo de Alajuela, Antonio del Carmen Monestel para posteriormente

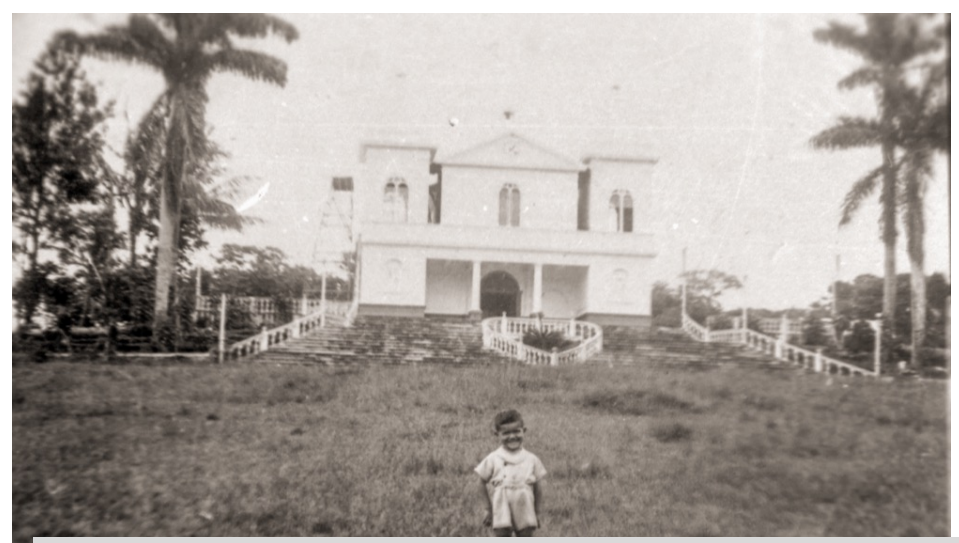

Foto 2 Santuario del Pilar en Zaragoza de Palmares en la década de 1970

Fuente: Anibal Arias. Colección personal. Palmares.

recurrir al arzobispo de San José, el alemán Juan Gaspar Stork para que se dirimiera el asunto. Al no encontrar una solución concreta y con motivo de una visita ad liminae a la Ciudad del Vaticano en 1905, monseñor Stork presentó la querella ante una comisión especial. Es presumible que los viejos altercados entre el clero y los liberales resonaban todavía, pues, se elevó un conflicto local a las más altas esferas de la Iglesia católica. Ahora bien, para los intereses de la Pilarica, el fallo posterior fue favorable, pese a ello y sin importar que proviniera de la misma Roma, Gómez se valió de diversas estrategias para maniobrar la vía del retorno de los fondos y de esa manera, pudo retardar la construcción del santuario mencionado por varios años. Una carta del sacerdote Mardoqueo Arce a su pastor Antonio del Carmen Monestel con fecha del 29 de agosto de 1921, ofrece una 
perspectiva del litigio, en razón de que todavía era comentado en la correspondencia y en otros documentos de la década de 1920 (Parroquia de la Merced de Palmares, 1921, s.p).

\section{Las luchas y el anhelo del pueblo de Zaragoza}

El templo parroquial estaba casi listo en 1914, sin embargo, en ese año estalló la Primera Guerra Mundial, que irremediablemente afectó las importaciones de las restantes estructuras metálicas, del cemento

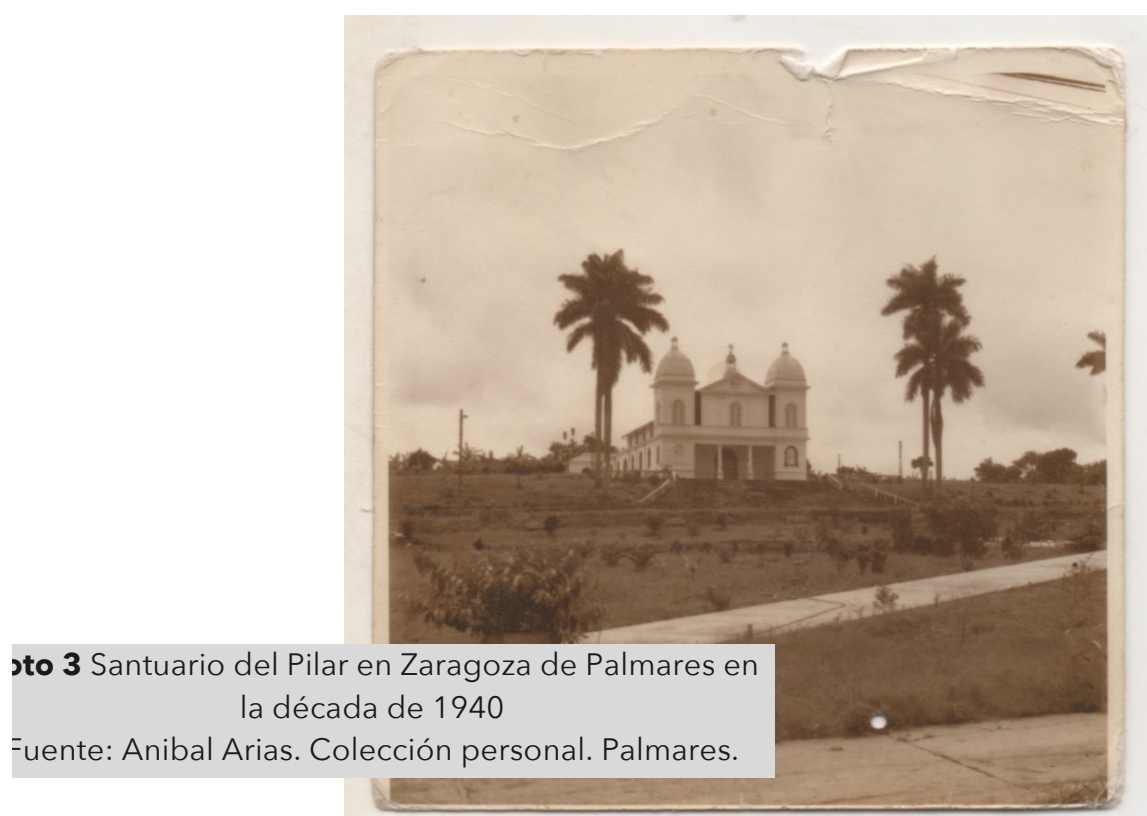

y de otros materiales importados desde Alemania, Bélgica o España. Una excusa más que posiblemente, se esgrimiría en detrimento de cualquier obra de infraestructura religiosa, mientras, Gómez mantuvo una actitud infranqueable hasta 1920, cuando se retiró de la Parroquia. Con el arribo del sacerdote Mardoqueo Arce, los ánimos cambiaron, más aún, este permitió la conformación de la primera Junta Edificadora de Zaragoza en agosto de 1921, tal como se consigna en el primer libro de actas. Por tanto, se deduce que la naciente Junta, se enfocaría en el proyecto frustrado de la ermita del Pilar, en todo caso, la sede parroquial no quedaba expuesta ni se ponía en riesgo sus intereses de cara al templo principal que ya se encontraba en pie.

En abril de 1922 arrancó el proyecto del santuario limpiando el área del terreno con la ayuda de los boyeros y luego cavando los cimientos en los que participaban de diversas formas los hombres, las mujeres y los menores. La tarea del levantamiento de las paredes de bahareque, las dos torres principales de cemento armado y el posterior techado, ya eran visibles en 1925, una razón de fondo para regocijarse y, por qué no, una victoria de los vecinos frente a un añejo litigio parroquial. La inconclusa, aunque, palpable obra envalentonó a la Junta 
Edificadora para decretar la celebración de la primera fiesta del Pilar al igual que la de Aragón en España, el mismo doce de octubre (La Tribuna, 1926). Es útil adicionar con el apoyo de los datos del Censo de Población de 1927, que Zaragoza contabilizó 1603 habitantes y el resto del cantón 6.683 , ello brindaría una perspectiva de las modestas dimensiones del poblado en relación con sus pretensiones, que le dieron un tipo de identidad comunal.

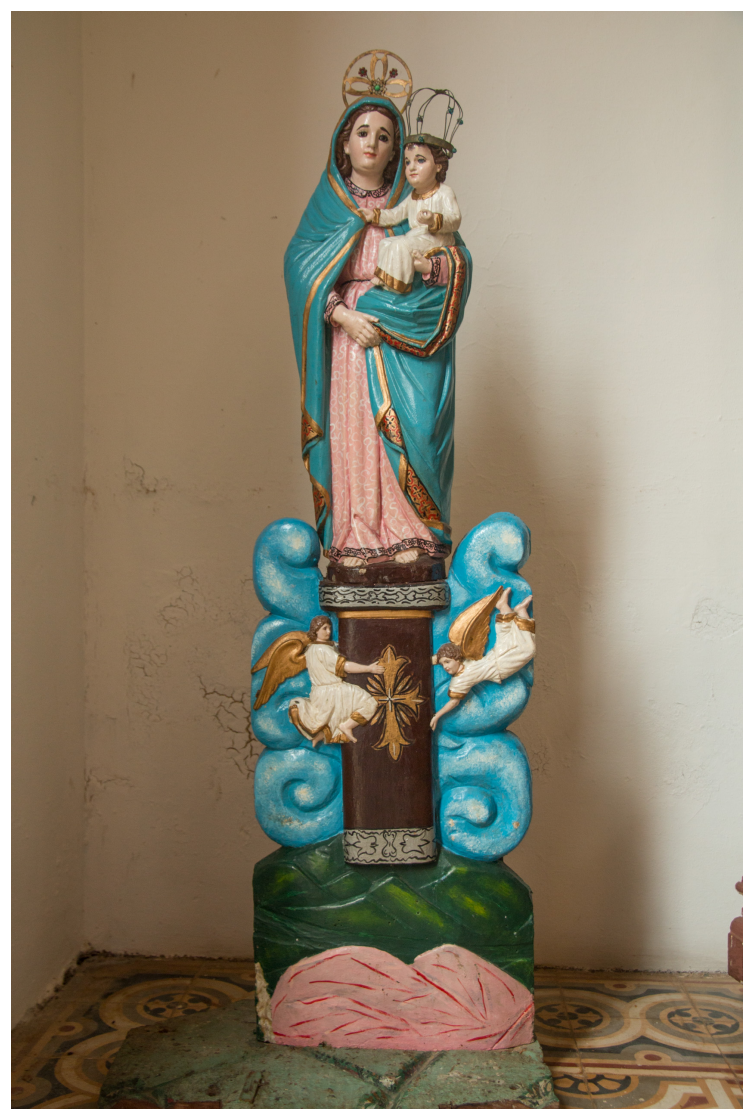

En la década de 1930, se pudo importar gracias al financiamiento de las mismas familias adineradas de la comunidad, los veintitrés vitrales fabricados en la ciudad de Barcelona, los cuales, rellenarían las mismas cavidades de las paredes laterales, de las dos torres frontales y del presbiterio, tal como se había previsto desde un inicio. Un aspecto que no debe dejarse por fuera, es que el imaginero religioso Manuel "Lico" Rodríguez labró en cedro una réplica del Pilar a finales del siglo XIX, que años más tarde,

Foto 4 Imagen de la Virgen del Pilar tallada por "Lico" Rodríguez en la década de 1890 Fuente: Juan Gabriel Madrigal. Colección personal. Palmares presumiblemente, fue donada por el señor Bruno Rojas al templo de Zaragoza para que fuera instalada en el altar mayor. ${ }^{4}$

\footnotetext{
${ }^{4}$ En un artículo mencioné que la imagen fue tallada por Bruno Rojas de San Mateo, una apreciación personal que era incorrecta, tal como me lo hizo saber el restaurador e imaginero ramonense Andrés Rodríguez Arias y que aclaro ahora. Pueden leer el error en Rodríguez Sancho (2007, p.147). El señor Manuel "Lico" Rodríguez murió en San Ramón en 1901, sus diversas esculturas con motivos de santos permanecen en varios templos católicos de la región de Occidente y en otros sitios. Se recomienda consultar el libro de Reymundo Méndez. (1997). Lico Rodríguez. Escultor de imaginería religiosa. San José: EUNED.
} 
A finales de la década de 1950, un ciudadano español de nombre Juan Manuel Bernal Alonso de paso por Palmares visitó la ermita de Zaragoza y de regreso a su natal España, propuso al ayuntamiento de Zaragoza el envío de una réplica para este pueblo rural costarricense. El arribo de la réplica provocó una nueva polémica, en esa ocasión, el

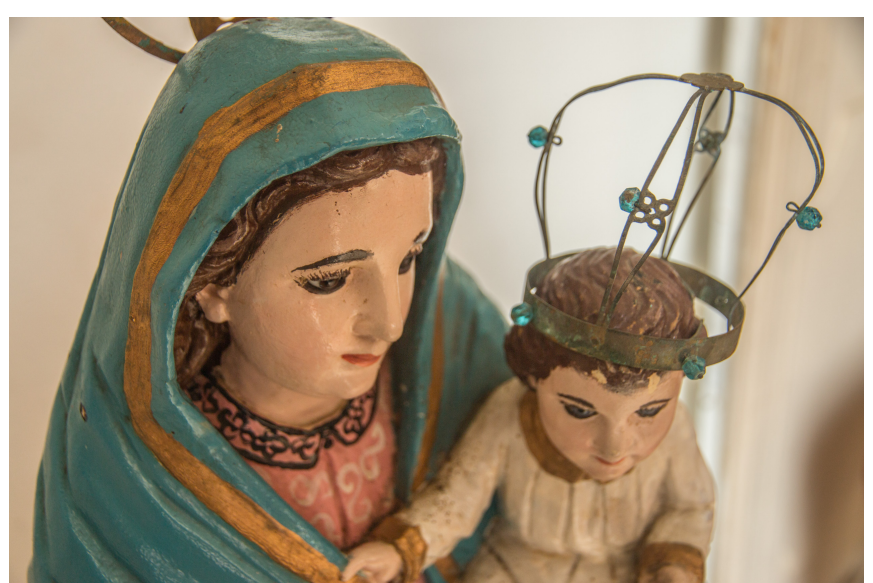

Foto 5 Detalle del rostro de la imagen de la Virgen del Pilar con el niño de "Lico" Rodríguez de la década de 1890

Fuente: Juan Gabriel Madrigal. Colección personal. cura español Venancio Oña Martínez destacado en la Parroquia, tuvo un altercado con los vecinos del caserío, quienes, le reclamaron con insistencia la entrega de la imagen que mantuvo durante varios días en la Casa Cural. El domingo 5 de junio de 1960 fue la fecha pactada por el Padre Oña para entregarla. Con base en el testimonio oral de algunos asistentes al evento, se aseguraba que de la Diócesis de Alajuela no hubo representación, un asunto que poco importó a los destinatarios locales del regalo religioso. Meses después, una comitiva de la Embajada de España acreditada en San José, entre ellos, el embajador Valentín Vía y Ventalló, asistieron a un festejo popular por invitación de la Junta Edificadora de Zaragoza que orgullosa quería mostrarse ante las autoridades diplomáticas, el cura o ante los habitante del cantón. (Junta Edificadora de Zaragoza, 1960, s.p). La réplica del Pilar de la Foto 1, es la enviada desde España y permanece en el altar mayor del santuario, esta sustituyó a la labrada por "Lico" Rodríguez en la Foto 4. Además, la Foto 2 es una toma exterior del santuario a comienzos de la década de 1970.

Por último, se reconoce que las fiestas patronales, los turnos $u$ otras celebraciones propias de los pueblos de la región central de Costa Rica y de otras zonas, albergan una importancia histórica que trasciende la vida de las pequeñas comunidades, muy a pesar de los altercados que hayan ocurrido entre las autoridades eclesiásticas, las civiles y las organizaciones locales. Para las comunidades, la celebración religiosa y popular guardaría un sentido simbólico y en 
esa medida, se ha forjado un tipo de identidad comunal. En este escenario, los vecinos de Zaragoza no son la excepción y conservan una memoria colectiva que se comenzó a fraguar desde el siglo XIX y con la celebración de la primera fiesta patronal del Pilar en octubre de 1925, se impulsó un sentido de pertenencia preservado por generaciones.

\section{BIBLIOGRAFÍA}

Abarca Vázquez, C. (1999). Siglo y medio de identidades palmareñas. San José: CAAV.

Archivo Eclesial de la Curia Metropolitana. (1906). Fondos Antiguos, caja 456. San José: Archivo Histórico Arquidiocesano. Arquidiócesis de San José, julio, (s.f).

Archivo Eclesial de la Curia Metropolitana. (1906). Fondos Antiguos, sub fondo, caja 33, folder 8. San José: Archivo Histórico Arquidiocesano. Arquidiócesis de San José, junio, folios 1-23.

Badilla, M \& Solórzano, W. (2010). De territorio a región. Bases estructurales para la creación de las regiones Occidente y Norte de Costa Rica: 1821-1955. San José: Sociedad Editora Alquimia 2000 S.A.

Cal, J. (2005). Iglesia y reformismo liberal en Guatemala y Costa Rica: siglo XIX. San José: Universidad de Costa Rica. Centro de Investigaciones Históricas de América Central, agosto.

Cedeño Castro, R. (2004). Religión civil o religión de Estado: el conflicto durante la reforma liberal en Guatemala y Costa Rica. Heredia: UNA. Departamento de Filosofía. Cuaderno Prometeo 31.

Censo de Población de Costa Rica. (1927). Censo de Población de 1927. San José: República de Costa Rica.

González Ortega, A. (1997). Vida cotidiana en la Costa Rica del siglo XIX. Un estudio psicogenético. San José: EUCR. 
Junta Edificadora de Zaragoza. (1921). Libro principal de actas: 1921 1947. Zaragoza de Palmares. Alajuela. Parroquia de la Merced de Palmares.

Junta Edificadora de Zaragoza. (1960). Libro principal de actas: 1959 y 1960. Zaragoza de Palmares. Alajuela. Parroquia de la Merced de Palmares.

La Tribuna. (1926, 10 de 0ctubre). La fiesta patronal de Zaragoza de Palmares. San José, (s.p).

Méndez Montero, R. (1997). Lico Rodríguez. Escultor de imaginería religiosa. San José: EUNED.

Parroquia de la Merced de Palmares. (1921). Carta de Mardoqueo Arce al obispo de Alajuela. Palmares. Diócesis de Alajuela, 29 de agosto.

Parroquia de la Merced de Palmares. (1904). Libro de actas de bautismo: 1900-1904, número IV. Palmares. Diócesis de Alajuela.

Parroquia de la Merced de Palmares. (1894-1914). Libros principales y actas de la parroquia. Palmares. Diócesis de Alajuela.

Pérez, M \& González, Y. (1993). "Iglesia y poder político. Un fragmento de historia comunal. Palmares 1866-1920", Revista de Ciencias Sociales (61): 97-113.

Pineda, M \& Castro, S. (2012). Colonización, poblamiento y economía. San Ramón: 1842-1900. $2^{\circ}$ edición. San José y San Ramón: Centro de Investigaciones Históricas. Sede de Occidente y Museo Regional de San Ramón.

Rodríguez Sancho, J. (2014). "Los boyeros en el noroccidente del Valle Central de Costa Rica entre los siglo XIX y XX", Diálogos Digital (volumen especial, octubre): 19-38.

Rodríguez Sancho, J. (2007). "Un legado histórico social y arquitectónico en el cantón de Palmares", Reflexiones, 86 (1): 141-150. 
Sandí Morales, J. (2011). "La participación de la Iglesia católica en el control del espacio en medio de la creación de un país llamado Costa Rica", Revista de Historia, 63 y 64 (enero- diciembre): 53-99.

Sanou, O. (2001). Arquitectura e historia en Costa Rica. Templos parroquiales en el Valle Central. Grecia, San Ramón y Palmares, 18601914. San José: EUCR.

Thiel, B.A. (1900). Cuadragésimo sexta carta pastoral. Para el fin del siglo XIX y principio del XX. San José: Tipografía de San José.

Torres, J.L. (2007). Naranjo y su historia: 1835-2004. San José: EUNED.

Vargas, C. (2015). Hacia la consolidación del Estado liberal en Costa Rica: 1870-1890. $2^{\circ}$ edición. San José: EUCR. Cuaderno de Historia de las Instituciones 27 .

Vásquez, R, Fallas, J \& Jiménez, R. (2014). La tumba de Colina Santiago, evidencias del contacto y post contacto en San Ramón de Alajuela, Costa Rica. San José: Museo Nacional de Costa Rica. Departamento de Antropología e Historia. Informe de rescate Arqueología 2014-086. 